\title{
Comparison of Proximal Femoral Nail Antirotation and Dynamic Hip Screw Internal Fixation on Serum Markers in Elderly Patients with Intertrochanteric Fractures
}

\author{
Bin Wang1, Qingbai Liu², Yu Liu³ and Rui Jiang²
}

\begin{abstract}
Objective: To compare effect of proximal femoral nail antirotation (PFNA) and dynamic hip screw (DHS) internal fixation on serum inflammatory mediators (CRP, IL-1, IL-6 and TNF- $\alpha$ ), myocardial injury markers (cTnT, CK-MB), and Myo-heart failure marker (BNP) in elderly patients with intertrochanteric fractures.

Study Design: Experimental study.

Place and Duration of Study: Department of Orthopedics, The Second Affiliated Hospital of Xuzhou Medical University, China, from January 2016 to February 2018.

Methodology: A total of 114 patients with intertrochanteric fractures were randomly divided into Group A and Group B, with 57 cases in each group. Group A was treated with PFNA and Group B with DHS internal fixation. Serum CRP, IL-1, IL-6, TNF- $\alpha$, CTnT, CK-MB, Myo, BNP and surgical indication were compared.

Results: Operation time and weight-bearing time in Group A were shorter than Group B (both $p<0.001$ ). Intraoperative blood loss and postoperative drainage volume in Group A were lower than Group B (both $p<0.001)$. On the $7^{\text {th }}$ day after surgery, serum CRP, IL-1, IL-6, TNF- $\alpha$, cTnT, CK-MB, Myo and BNP in Group A were lower than Group B (all $p<0.001$ ).

Conclusion: Compared with DHS, PFNA effectively reduced serum inflammatory mediators with less damage to cardiac function and myocardium in elderly patients with intertrochanteric fractures.
\end{abstract}

Key Words: Elderly, Femur, Intertrochanteric fracture, Intramedullary nail, Dynamic hip screw (DHS).

\section{INTRODUCTION}

Intertrochanteric fracture occurs below the hip capsule line of the femoral neck base and above the level of the lesser trochanter. It occurs mostly in the elderly, and is more commonly seen in women than men. Accounting for about half of hip fractures, it is one of the common injuries in the elderly.1,2 At present, non-surgical and surgical treatment is often used clinically in elderly patients with intertrochanteric fractures. ${ }^{3}$ Non-surgical treatment requires long-term bed rest, and subsequent complications such as hypostatic pneumonia, urinary tract infections, muscle atrophy, and joint stiffness, which can exert a serious impact on the prognosis of patients and the quality of life in the future.

Internal fixation is a common surgical method for the treatment of intertrochanteric fractures in the elderly. It is

1 Department of Orthopedics, The Second Affiliated Hospital of Xuzhou Medical University, 221006, China

2 Department of Orthopedics, Lianshui County People's Hospital, Huai'an, 223400, China

3 Department of Orthopedics, Wuxi Orthopaedic Hospital, 214062, China

Correspondence: Rui Jiang, Department of Orthopedics, Lianshui County People's Hospital, Huai'an, 223400, China E-mail: qianyou699@163.com

Received: October 09, 2018; Revised: January 01, 2019; Accepted: January 10, 2019 generally divided into intramedullary fixation and extramedullary fixation. Intramedullary fixation mainly includes proximal femoral nail (PFN), proximal femoral nail antirotation (PFNA), etc.; extramedullary fixation mainly includes locking plate and DHS. 4 Among them, PFNA is mainly developed for elderly patients with osteoporosis. ${ }^{5}$ It is the method of choice for surgical treatment of unstable inter-trochanteric femoral fractures. 6 DHS, designed by AO/ASIF (Association for the Study of Internal Fixation), is a fixation method for proximal and distal femoral fractures. ${ }^{7}$ Compared with the Holland nail, DHS could be implanted more quickly and with less exposure to radiation, but the resultant blood loss and need for transfusion is greater. 8

CRP, IL-1, IL-6 and TNF- $\alpha$ are common serum inflammatory mediators. CRP is a systemic inflammatory marker regulated by cytokines such as IL-1, IL- 6 , and TNF- $\alpha .9$ They are all involved in the occurrence, development of fractures and increase bone resorption. In elderly patients with intertrochanteric fractures, due to anesthesia, stress response caused by surgical trauma, circulatory capacity changes and water and sodium retention occurs during the perioperative period, the patients may undergo adverse changes in cardiovascular function, and even myocardial infarction and heart failure may be induced, bringing difficulties in clinical treatment. Cardiac troponin $\mathrm{T}$ (cTnT), creatine kinase isoenzyme (CK-MB), and myoglobin (Myo) are 
three common biochemical markers of myocardial infarction. Currently recognised as important markers of myocardial injury, they are of great significance for early detection of myocardial infarction. 10 B-type natriuretic peptide (BNP) is an important biomarker for heart failure diagnosis and risk stratification. ${ }^{11}$ At present, there are few studies on early warning of postoperative myocardial infarction and heart failure in elderly patients with intertrochanteric fractures. The research on PFNA and DHS for the treatment of intertrochanteric fractures is limited to the observation of basic curative effect, and there is a lack of comparative analysis from serum inflammatory mediators, myocardial injury markers and heart failure markers.

The objective of this study was to compare effect of PFNA and DHS internal fixation on the serum levels of inflammatory mediators like CRP, IL-1, IL-6 and TNF- $\alpha$; myocardial injury markers like cTnT, CK-MB; and Myo and heart failure marker like BNP in elderly patients with intertrochanteric fractures.

\section{METHODOLOGY}

This study was conducted at Department of Orthopedics, The Second Affiliated Hospital of Xuzhou Medical University, China, from January 2016 to February 2018. Approved by the Ethics Committee of the Hospital, all patients volunteered to participate in the study. A total of 114 elderly patients with intertrochanteric fractures were selected as subjects. Inclusion criteria were all patients diagnosed with intertrochanteric fractures on X-ray; no cardiac accidents such as angina pectoris, myocardial infarction and heart failure within 6 months before admission; no cognitive disorder; and preoperative blood pressure and blood sugar; and other common diseases were controlled in a normal state. Exclusion criteria were severe cardiovascular and cerebrovascular disease; patients combined with other fractures; pathological hip fractures; mental illness before fracture; and patients with surgical contraindications.

Patients were divided into Group A and Group B according to the random number table method, with 57 cases in each group. Group A was treated with PFNA. Patient was placed in the supine position. Epidural anesthesia was given, and closed reduction of the fracture was performed under C-arm fluoroscopy. Then the incision was cut vertically $5 \mathrm{~cm}$ above the apex of the greater trochanter. A curved opener was used to open the apex of the greater trochanter. The guide pin was inserted in the center of the medullary cavity in a positive lateral position under the C-arm machine fluoroscopy. The proximal end of the guide pin was used to define the reaming. The main nail was inserted into the proper position, and the proximal aiming arm was used to insert the guide needle into the femoral neck. The position of the guide needle was checked by C-arm fluoroscopy. The length was measured. The outer cortex of the femoral trochanter was opened with a hollow drill bit, and the spiral blade was struck along the direction of the guide needle. The position of the spiral blade was checked with $\mathrm{C}$-arm machine again. When the length was appropriate, the spiral blade was locked, and then the distal locking pin was locked under the guidance of the aiming arm. After the operation, the incision was washed and closed layer by layer after the negative pressure drainage was placed.

Group B was treated with DHS internal fixation. Patient was placed in the supine position. Continuous epidural anesthesia was used, and the affected side of the hip was raised. After the patient was reset to a satisfactory degree with the C-arm machine, a $10-15 \mathrm{~cm}$ lateral incision of the hip joint was cut to separate and reveal the greater trochanter and the upper end of the lateral femora. The $135^{\circ}$ guider was first used next to the upper end of the lateral femoral cortex for inserting compression screw guide needle of the femoral neck. After adjusting the guide needle to a satisfactory position in a positive axial position under the $\mathrm{C}$-arm machine fluoroscopy, femoral neck was reamed. The depth was measured. The compression screw was screwed. Then the position of the main screw was checked in a positive lateral position under the C-arm machine fluoroscopy. After the length was appropriate, the side plate was inserted finally, ensuring that the steel plate was located on the longitudinal axis of the femur next to the lateral cortical bone. Then the screws were used for fixation.

Both groups were routinely treated with antibiotics for anti-infective treatment. The drainage tube was routinely placed for 48 hours, and the amount, color and traits of the drainage fluid were recorded. On the 2 nd day after surgery, the patient was allowed to sit half-squat, sit up, turn over, and perform contractile function exercise of the active and passive muscle, as well as knee flexion and extension exercises.

The surgical indications such as operation time, intraoperative blood loss, postoperative drainage volume and weight-bearing time were compared after operation. The blood of the elbow vein was taken from patients in the two groups, the day before operation and on the $7^{\text {th }}$ day after operation. The serum levels of inflammatory mediators CRP, IL-1, IL- 6 and TNF- $\alpha$ were detected by ELISA. Roche electrochemiluminescence analyser was used to measure and compare the changes of serum myocardial injury markers cTnT, CK-MB and Myo, and the levels of heart failure marker BNF in the two groups.

SPSS 23.0 software was adopted for data statistical analysis. Mean value $\pm S D$ was calculated for measurement data, and examined by independent sample t-test. Enumeration data was represented by $\mathrm{n}(\%)$, and examined by $\chi^{2}$ test. The $p<0.05$ means there was statistical significance in the difference. 


\section{RESULTS}

Among 114 patients, 43 were males $(37.72 \%)$ and 71 females $(62.28 \%)$. They were aged $65-83$ years, with an average age of $73.16 \pm 3.47$ years. The time from injury to surgery was 2-8 days, with an average of $4.18 \pm 0.72$ days. According to Evans-Jensen classification: 33 cases $(28.95 \%)$ of type I, 41 cases $(35.96 \%)$ of type II, 32 cases $(28.07 \%)$ of type III, and 8 cases $(7.02 \%)$ of type IV, all of which were closed fractures. Fifty cases $(43.86 \%)$ had hypertension and 41 cases $(35.96 \%)$ had diabetes.

The operation time and weight-bearing time of Group $A$ were shorter than those of Group B (both $p<0.001$ ). The intraoperative blood loss and postoperative drainage volume in Group A were less than those in Group B (both $\mathrm{p}<0.001$, Table I).

There were no significant differences in the serum levels of CRP, IL-1, IL- 6 and TNF- $\alpha$ between the two groups $(p=0.965,0.867,0.893$ and 0.977 , respectively) before

Table I: Comparison of surgical indications.

\begin{tabular}{l|c|c|c}
\hline Parameters & $\begin{array}{c}\text { Group A } \\
(\text { Mean } \pm \text { SD }) \\
\mathrm{n}=57\end{array}$ & $\begin{array}{c}\text { Group B } \\
(\text { Mean } \pm \text { SD }) \\
\mathrm{n}=57\end{array}$ & -value \\
\hline $\begin{array}{l}\text { Operation time } \\
\text { (minutes) } \mathrm{n}=57\end{array}$ & $70.56 \pm 5.32$ & $86.24 \pm 6.71$ & $<0.001$ \\
\hline $\begin{array}{l}\text { Intraoperative blood loss } \\
(\mathrm{mL}) \mathrm{n}=57\end{array}$ & $214.78 \pm 14.93$ & $302.45 \pm 18.04$ & $<0.001$ \\
\hline $\begin{array}{l}\text { Postoperative drainage volume } \\
(\mathrm{mL}) \mathrm{n}=57\end{array}$ & $92.63 \pm 8.55$ & $113.91 \pm 13.02$ & $<0.001$ \\
\hline $\begin{array}{l}\text { Weight-bearing time } \\
(\text { week }) \mathrm{n}=57\end{array}$ & $5.82 \pm 1.07$ & $7.76 \pm 1.45$ & $<0.001$ \\
\hline
\end{tabular}

$S D=$ Standard deviation surgery. On the 7 th day after surgery, serum levels of CRP, IL-1, IL-6 and TNF- $\alpha$ in Group A were lower than Group B (all $p<0$. 001, Table II).

There were no significant differences in the serum levels of myocardial injury markers CTnT, CK-MB and Myo and the heart failure marker BNP $(p=0.509,0.917,0.972$ and 0.955 , respectively) between the two groups before surgery; on the $7^{\text {th }}$ day after surgery, the serum levels of cTnT, CK-MB, Myo and BNP in Group A were lower than Group B (all $p<0$. 001, Table III).

\section{DISCUSSION}

In clinical practice, DHS has been found to have certain disadvantages such as large cortical lag screws that are easy to damage the lateral wall of the femur during the operation and early stage activities, resulting in an increase in fracture damage. There is also great damage to blood supply, non-healing, delayed healing and other complications after DHS operation.12,13 As an intramedullary fixation system, PFNA overcomes the shortcomings of DHS with a better designed procedure. There are many advantages of PFNA. It can withstand large axial shearing force, and the spiral blade strengthens the fixation of the femoral neck and femoral head. The spiral blade combined with the distal fixation screw avoids the rotation of the main nail, which makes patients able to carry out weight-bearing exercise quickly after surgery. There is little trauma without lateral exfoliation of the femoral muscle during surgery, which is important for maintaining the stability of the lateral femur; and because of the use of single spiral blade in surgery. The stability is improved while avoiding the screw

Table II: Comparison of serum inflammatory factors.

\begin{tabular}{|c|c|c|c|c|}
\hline Indices & Time & $\begin{array}{c}\text { Group A (Mean } \pm \text { SD) } \\
\qquad \mathrm{n}=57\end{array}$ & $\begin{array}{c}\text { Group B (Mean } \pm \text { SD) } \\
n=57\end{array}$ & $p$-value \\
\hline CRP (mg/L) & Before surgery & $11.36 \pm 4.57$ & $11.32 \pm 5.03$ & 0.965 \\
\hline $\mathrm{n}=57$ & On the $7^{\text {th }}$ day after surgery & $12.73 \pm 2.94$ & $14.73 \pm 2.43$ & $<0.001$ \\
\hline IL-1 (ng/mL) & Before surgery & $13.14 \pm 1.85$ & $13.08 \pm 1.96$ & 0.867 \\
\hline $\mathrm{n}=57$ & On the $7^{\text {th }}$ day after surgery & $14.25 \pm 2.02$ & $16.33 \pm 2.75$ & $<0.001$ \\
\hline $\mathrm{IL}-6(\mu \mathrm{g} / \mathrm{mL})$ & Before surgery & $43.58 \pm 4.65$ & $43.47 \pm 4.02$ & 0.893 \\
\hline $\mathrm{n}=57$ & On the $7^{\text {th }}$ day after surgery & $44.76 \pm 3.51$ & $50.86 \pm 5.19$ & $<0.001$ \\
\hline TNF- $\alpha(n g / m L)$ & Before surgery & $448.93 \pm 35.16$ & $448.75 \pm 30.22$ & 0.977 \\
\hline $\mathrm{n}=57$ & On the $7^{\text {th }}$ day after surgery & $471.72 \pm 28.93$ & $512.36 \pm 34.08$ & $<0.001$ \\
\hline
\end{tabular}

SD = Standard deviation.

Table III: Comparison of the serum levels of myocardial injury markers and heart failure markers.

\begin{tabular}{|c|c|c|c|c|}
\hline Indices & Time & $\begin{array}{c}\text { Group A (Mean } \pm \text { SD) } \\
\qquad n=57\end{array}$ & $\begin{array}{l}\text { Group B (Mean } \pm \text { SD) } \\
\qquad n=57\end{array}$ & $p$-value \\
\hline cTnT (ng/mL) & Before surgery & $0.15 \pm 0.07$ & $0.14 \pm 0.09$ & 0.509 \\
\hline$n=57$ & On the $7^{\text {th }}$ day after surgery & $0.54 \pm 0.21$ & $0.75 \pm 0.37$ & $<0.001$ \\
\hline CK-MB (ng/mL) & Before surgery & $2.42 \pm 1.65$ & $2.39 \pm 1.42$ & 0.917 \\
\hline $\mathrm{n}=57$ & On the $7^{\text {th }}$ day after surgery & $3.26 \pm 1.83$ & $4.40 \pm 1.11$ & $<0.001$ \\
\hline Myo (ng/mL) & Before surgery & $84.85 \pm 9.37$ & $84.79 \pm 8.66$ & 0.972 \\
\hline$n=57$ & On the $7^{\text {th }}$ day after surgery & $112.63 \pm 5.92$ & $123.31 \pm 6.74$ & $<0.001$ \\
\hline $\mathrm{BNP}(\mathrm{pg} / \mathrm{mL})$ & Before surgery & $59.75 \pm 6.04$ & $59.68 \pm 7.13$ & 0.955 \\
\hline$n=57$ & On the $7^{\text {th }}$ day after surgery & $71.08 \pm 4.15$ & $83.44 \pm 8.21$ & $<0.001$ \\
\hline
\end{tabular}

SD = Standard deviation 
loosening or withdrawal. At the same time, close reduction can be performed with percutaneous internal fixation, shortening the operation time and reducing the trauma. 14

This study found that compared with DHS, PFNA treating intertrochanteric fractures in the elderly enjoyed the advantages of smaller surgical trauma, shortened operation time and less amount of surgical bleeding and drainage. This is basically consistent with previous research reports. ${ }^{15}$ In this study, patients treated with DHS had a weight-bearing time later than those treated with PFNA. This may be due to the fact that DHS fracture fixation is weaker than PFNA, so patients with DHS are often told to avoid full weight bearing. ${ }^{16}$

When a patient's body is traumatised, the serum inflammatory factors change more obviously due to the traumatic stress. The serum levels of CRP, IL-1, IL- 6 and TNF- $\alpha$ can reflect the degree of stress in the body. ${ }^{17,18}$ This study found that compared with DHS, PFNA can effectively reduce the serum level of inflammatory mediators, which is conducive to the recovery of patients' body.

At present, not enough importance is attached to the risk of early myocardial infarction and heart failure in elderly patients with intertrochanteric fractures. Relevant examinations and treatments are often performed after the obvious symptoms of myocardial infarction or heart failure, leading to a delay in treatment. CTnT is widely used in the clinical detection of myocardial injury and unstable angina. Sandhu et al. found that CTnT had a positive prognostic effect on the morbidity and mortality of surgical treatment of elderly patients with hip fracture. ${ }^{19}$ Chong et al. showed that troponin was a prognostic indicator of cardiac complications after orthopedic surgery. ${ }^{20}$ Myo is an early indicator of myocardial infarction and is generally used to assess complications and reinfarction in patients. CK-MB is an important indicator for the diagnosis of acute myocardial infarction and the presence or absence of myocardial infarction. CTnT, Myo and CK-MB are currently recognised as good markers of myocardial injury, and are of great significance for the diagnosis of myocardial infarction and its sudden death. BNP test can be used to distinguish between dyspnea caused by heart failure and water and sodium retention, as well as provide some warning signs for heart failure or other heart diseases. Currently, BNP has been used as an independent diagnostic criterion for heart failure clinically. ${ }^{21}$ The study indicated that PFNA and DHS surgeries had a certain degree of adverse effects on cardiac function in elderly patients with intertrochanteric fractures; but compared with DHS, PFNA had less damage to cardiac function and myocardium.

Because of the small sample size in this study, there are many influencing factors to be further considered, such as basic diseases, gender, type of fracture, surgical procedure and length of postoperative follow-up. The effects of PFNA and DHS on biochemical indicators such as cTnT, CK-MB, Myo and BNP still need follow-up of large-scale clinical cases to better guide clinical practice.

\section{CONCLUSION}

Compared with DHS, PFNA effectively reduced serum inflammatory mediators with less damage to cardiac function and myocardium in elderly patients.

\section{REFERENCES}

1. Ehlers MM, Nielsen CV, Bjerrum MB. Experiences of older adults after hip fracture: An integrative review. Rehabil Nurs 2018; 43:255-66.

2. Joshi D, Dhamangaonkar AC, Ramawat S, Goregaonkar AB. Predictors of iatrogenic lateral wall fractures while treating intertrochanteric fracture femur with the dynamic hip screw system in Indian patients. Eur J Orthop Surg Traumatol 2014; 25:677-82.

3. Nasab SAM, Khorramdin E. The assessment of mortality and quality of life after intertrochanteric fracture of femur in patients older than 60 at Emam Khomeini Hospital of Ahvaz. Pak J Med Sci 2017; 33:895-8.

4. Pradeep AR, KiranKumar A, Dheenadhayalan J, Rajasekaran S. Intraoperative lateral wall fractures during dynamic hip screw fixation for intertrochanteric fractures - Incidence, causative factors and clinical outcome. Injury 2017; 49:334-8.

5. Cho HM, Lee K. Clinical and functional outcomes of treatment for type A1 intertrochanteric femoral fracture in elderly patients: Comparison of dynamic hip screw and proximal femoral nail antirotation. Hip Pelvis 2016; 28:232-42.

6. Sahin S, Ertürer E, Oztürk I, Toker S, Seçkin F, Akman S. Radiographic and functional results of osteosynthesis using the proximal femoral nail antirotation (PFNA) in the treatment of unstable intertrochanteric femoral fractures. Acta Orthop Traumatol Turc 2010; 44:127-34.

7. Sharma A, Sethi A, Sharma S. Treatment of stable intertrochanteric fractures of the femur with proximal femoral nail versus dynamic hip screw: A comparative study. Rev Bras Ortop 2017; 53:477-81.

8. Little NJ, Verma V, Fernando C, Elliott DS, Khaleel A. A prospective trial comparing the Holland nail with the dynamic hip screw in the treatment of intertrochanteric fractures of the hip. J Bone Joint Surg Br 2008; 90:1073-8.

9. Macêdo Santiago LÂ, Neto LGL, Borges Pereira G, Leite RD, Mostarda CT, de Oliveira Brito Monzani J, et al. Effects of resistance training on immunoinflammatory response, TNF-alpha gene expression, and body composition in elderly women. J Aging Res 2018; 2018:1467025.

10. Lan H, Du W, Mo Z, Huang H. The influence of blood collection tubes on measurement of cardiac biomarkers. Clin Lab 2016; 62:705-9.

11. Linssen GCM, Jaarsma T, Hillege HL, Voors AA, van Veldhuisen DJ. A comparison of the prognostic value of BNP versus NTproBNP after hospitalisation for heart failure. Neth Heart $J$ 2018; 26:486-92. 
12. Gashi YN, Elhadi AS, Elbushra IM. Outcome of primary cemented bipolar hemiarthroplasty compared with dynamic hip screw in elderly patients with unstable intertrochanteric fracture. Malays Orthop J 2018; 12:36-41.

13. Kweon SH, Lee SH, Kook SH, Choi YC. Outcomes of cephalomedullary nailing in basicervical fracture. Hip Pelvis $2017 ; 29: 270-6$

14. Sharma A, Mahajan A, John B. A comparison of the clinicoradiological outcomes with proximal femoral nail (PFN) and proximal femoral nail antirotation (PFNA) in fixation of unstable intertrochanteric fractures. J Clin Diagn Res 2017, 11:RC05-9.

15. Huang SG, Chen B, Zhang Y, Nie FF, Ju L, Li M, et al. Comparison of the clinical effectiveness of PFNA, PFLCP, and DHS in treatment of unstable intertrochanteric femoral fracture. Am J Ther 2017; 24:e659-66.

16. Temiz A, Durak A, Atici T. Unstable intertrochanteric femur fractures in geriatric patients treated with the DLT trochanteric nail. Injury 2015; 46:S41-6.
17. Kaiser K, Prystaz K, Vikman A, Haffner-Luntzer M, Bergdolt S, Strauss G, Waetzig GH, et al. Pharmacological inhibition of IL-6 trans-signaling improves compromised fracture healing after severe trauma. Naunyn Schmiedebergs Arch Pharmacol 2018; 391:523-36

18. Gong Y, Zhang Y, Tao S. Nalbuphine for analgesia after fracture surgery and its effect on circulating inflammatory factors. Exp Ther Med 2017; 15:859-63.

19. Sandhu A, Sanders S, Geraci SA. Prognostic value of cardiac troponins in elderly patients with hip fracture - a systematic review. Osteoporos Int 2013; 24:1145-9

20. Chong CP, van Gaal WJ, Savige J, Lim WK. Cardiac injury and troponin testing after orthopaedic surgery. Injury 2011; 42: 855-63.

21. Holzhauser L, Kim G, Sayer G, Uriel N. The effect of left ventricular assist device therapy on cardiac biomarkers: Implications for the identification of myocardial recovery. Curr Heart Fail Rep 2018; 15:250-9. 